This is the author's version of a work published in Phys. Rev. E 100, 013115, DOI: 10.1103/PHYSREVE.100.013115

\title{
Electrical conductivity and tortuosity of solid foam: effect of pore connections
}

\author{
V. Langlois* \\ Université Paris-Est, Laboratoire Géomatériaux et Environnement (EA 4508), UPEM, F-7ry454, Marne-la-Vallée, France \\ V.H. Trinh ${ }^{\dagger}$ \\ Le Quy Don Technical University, Hanoi, Vietnam \\ C. Perrot ${ }^{\ddagger}$ \\ Université Paris-Est, Laboratoire Modélisation et Simulation Multi échelle, \\ MSME UMR 8208 CNRS, Marne-la-Vallée 77454, France
}

\begin{abstract}
Numerical and analytical methods at both micro- and mesoscales are used to study how the electrical resistivity and the high frequency tortuosity of solid foam are modified by the presence of membranes that partially or totally close the cell windows connecting neighbor pores. Finite element method (FEM) simulations are performed on two pores connected by a single-holed membrane and on well-ordered Kelvin foam. For two pores connected by a single-holed membrane, we show that the equation for pore access resistance obtained by Sahu and Zwolak (Phys. Rev. E 98, 012404, 2018) can predict, after a few modifications, the electrical resistivity at the membrane scale for a large range of membrane apertures. In the second part, considering these analytical results, we build a pore-network model by using two kinds of conductances at the pore scale - inter-pore conductance and intra-pore conductance. Local inter-pore resistances govern foam electrical conductivity at small membrane aperture size, but when the membrane aperture has the same order of magnitude as the pore size, the intra-pore resistances are no longer negligible. An important success of this pore-network model is that it can be used to study the effects of percolation on the foam electrical conductivity by using pore-network simulations on larger samples containing a few thousands of pores and having different proportions of closed membrane randomly distributed over the sample. The tortuosity is found to be drastically larger than one in foam containing membranes with small apertures or a significant fraction of closed membranes.
\end{abstract}

\section{INTRODUCTION}

When an acoustic wave propagates through a viscous fluid-saturated porous media having a motionless skeleton (rigid frame), the relative displacement between fluid and frame generates fluid shear at the surface of the pore walls. This results in a viscous dissipation and an attenuation of the sound. Due to its diffusive propagation, any vorticity generated at the pore walls decays to zero as one moves away from the pore wall into the bulk of the pore [1]. Therefore, viscous dissipation occurs in a boundary layer located at the surface of the pore and of thickness $\delta$. This length $\delta$, called viscous skin depth, is frequency dependent: $\delta \approx \sqrt{\mu /\left(\rho_{f} f\right)}$ where $\mu$ and $\rho_{f}$ are respectively the dynamic viscosity and density of the fluid, and $f$ is the frequency. In the limit of high frequencies, the viscous skin depth becomes much smaller than any characteristic pore size, and the fluid tends to behave as an ideal fluid having no viscous effect (except in the thin boundary layer). More generally, the dynamic behavior of fluid-saturated porous media is due to a balance between the power developed by the fluid pressure gradient and both the viscous dissipative power and the inertial power developed in the fluid $[2,3]$. The

\footnotetext{
* vincent.langlois@u-pem.fr

$\dagger$ hai.tv@lqdtu.edu.vn

$\ddagger$ camille.perrot@u-pem.fr
}

viscous dissipative power dominates the inertial power at low frequency and inversely at high frequency. The visco-intertial frequency $f_{v}$ defining the transition between low- and high-frequency behaviour, corresponds to the frequency for which the viscous dissipated and kinetic (inertial) powers are equal [3]. Likewise, the distinction between the low- and high-frequency behaviour depends also on whether the viscous skin depth is large or small compared to a characteristic pore size [1]. An estimate of $f_{v}$ follows from this phenomenological description, and is given by $f_{v}=\mu /\left(K \alpha_{\infty} \rho_{f}\right)$; where $K$ is the permeability, a parameter associated with the low frequency behavior; $\alpha_{\infty}$ is the tortuosity, a parameter associated with the high-frequency behavior. In the high-frequency limit, the effective density $\rho_{e}$ only depends on the tortuosity, $\rho_{e}=\alpha_{\infty} \rho_{f}$.

The tortuosity is a key parameter describing dispersion of microscopic velocities with respect to the average value of microscopic velocities (Eq. A4). Because at high frequency, the potential flow is formally identical to electric conduction assuming that the porous solid is insulating [4], the tortuosity can be experimentally estimated by electrical conductivity measurement or calculated for specific pore geometries by solving the electrical boundary value problem (Appendix A, Sec. Inertial flow, Eq. A3). Several properties of $\alpha_{\infty}$ are important to mention. (1) $\alpha_{\infty}$ is a scale invariant parameter (i.e. remaining unchanged if the sample is uniformly dilated or contracted by a scale factor) and is greater than or equal to one. (2) 
$\alpha_{\infty}$ is dependent on the shape of the solid matrix, for example it accounts for both sinuosity and convergingdiverging pore geometry. (3) In the theory proposed by Biot, $\alpha_{\infty}$ is a "structure constant" defined as a purely geometric parameter, independent of material parameters, which relates an inertial fluid-solid coupling density $\rho_{a}$ to the density of the fluid filling the pore space $\rho_{f}$, $\rho_{a}=\left(\alpha_{\infty}-1\right) \rho_{f}[2,4,5]$. This coupling term is related to the force applied to a fluid to prevent its displacement when the solid is accelerated. (4) At high frequency, the effective sound speed is proportional to $1 / \sqrt{\alpha_{\infty}}$, meaning that the effective distance of travel for the wave between two points is increased by $\sqrt{\alpha_{\infty}}$ due to the tortuous path [6].

Foam is a dispersion of gas in a liquid or solid matrix. Its structure consists of membranes (also called films for liquid foams), ligaments or Plateau's borders (junction of three membranes) and vertices or nodes (junction of four ligaments). Whereas closed membranes are necessary to ensure the mechanical stability of liquid foam [7], they can be open or totally absent in solid foam allowing for the foam cells (pores) to be connected through windows. The closed membranes have drastic effects on transport phenomena, such as fluid flow or electric current: open windows participate in the transport through the material, whereas closed windows stop it. Therefore the fraction of closed windows is crucial for several applications (filtering or sound absorption). The effects of membrane on acoustical properties are now the subject of active research [3, 8-11]. It has been shown that closed membranes can increase the effective density of foam [8] and therefore, in agreement with the work of Jonhson et al. [1], the foam tortuosity $\alpha_{\infty}$ [10]. Moreover, closed membranes imply some percolation effects at the mesoscale which can drastically reduce the foam permeability $K$ as shown in [9].

Addressing the percolation issue of the electric conductivity of porous media requires numerical simulations with large samples involving a few thousand pores [12]. As the size of samples increases, the computational costs for the major numerical methods (finite element, finite volume and boundary element methods) become prohibitive, so that alternative approaches are preferable. Such methods involve determining the flow behavior at the local scale (i.e. a throat between two linked pores) by numerical simulations or analytical solutions (e.g. Hagen-Poiseuille equation), then pore-network simulations at the mesoscopic scale are performed [13, 14]. This intermediate scale between micro- and macro- scales is necessary to capture collective effects such as percolation behavior. Such a method was used by Johnson et al. to study the electrical conductivity of porous media. In their simple model, the pores are connected by tubes for which the electrical resistance is perfectly known. The electrical conductivity of the porous media is determined by calculating the equivalent resistance of the tube network. Moreover, it was recently shown that the porenetwork method can successfully predict the effects of closed membranes on foam permeability, and can be used to validate a model of effective permeability [9]. As the problem of foam electrical conductivity and the one of foam permeability share some similarities [15], it is appealing to check if the method used to estimate the effective permeability of foam could be transposed to compute the effective electric conductivity and the tortuosity of foam with membrane.

In this paper, we use a multi-scale approach to study the electrical conductivity of solid foam with various window configurations. Firstly, we deal with the case of two pores separated by a thin membrane having a circular hole. We calculate both the electrical conductance and the fluid flow conductance by using FEM. Then, we consider a more realistic pore geometry by using a Kelvin partition of space. We conduct FEM simulations on periodic unit cells (PUC) containing two interconnected pores with various open membrane fractions. The two previous steps are used to build a pore-network model able to reproduce the FEM results. Then, mesoscopic effects induced by the structure of the pore network are studied by pore-network simulations on large $(16 \times 16 \times 32$ pores) networks of electrical resistances.

\section{FLUID PERMEABILITY AND ELECTRICAL CONDUCTION IN POROUS MEDIA}

In this section, we recall the definition of the macroscopic parameters involved in this paper: permeability for the issue of Newtonian fluid flow through the pore space, and electrical conductivity for the issue of electrical current through a conducting fluid filling the pore space.

Permeability $K$ can be determined by measuring the rate of fluid flow $Q$ through a surface of porous media when the latter is subjected to a pressure difference $\Delta P_{s p}$ between its opposite faces[15, 16]:

$$
K=\left[\mu Q H_{s p}\right] /\left[A_{s p} \Delta P_{s p}\right]
$$

where $\mu$ is the dynamic viscosity of the fluid, $H_{s p}$ the thickness of the sample and $A_{s p}$ its cross-section area.

Effective electrical conductivity $\sigma_{e}$ is determined by measuring the total current $I$ passing through a surface of porous media when this one is subjected to an electrical potential difference $\Delta V_{s p}$ applied across the system:

$$
\sigma_{e}=\left[I H_{s p}\right] /\left[A_{s p} \Delta V_{s p}\right]
$$

As shown in [4], the high frequency tortuosity $\alpha_{\infty}$ is related to the electrical conductivity as follows:

$$
\frac{\phi}{\alpha_{\infty}}=\frac{\sigma_{e}}{\sigma_{f}}=\frac{1}{F}
$$




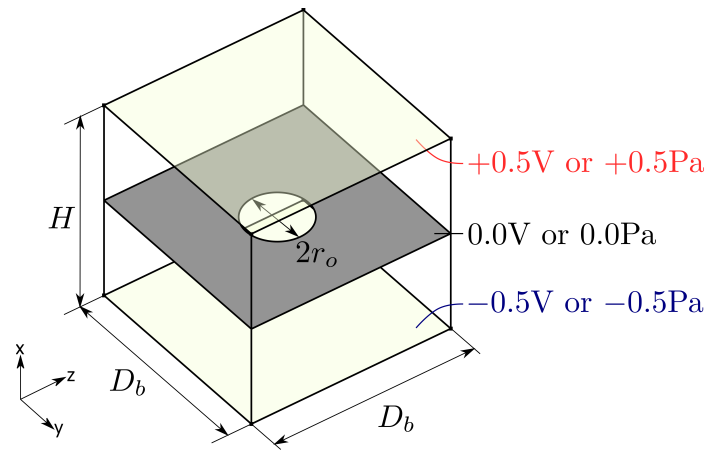

Figure 1. Two pores connected by a single perforated membrane. Depending on the physical problem under study, the boundary conditions are different. For the fluid flow case, a pressure drop is applied between the top and the bottom faces, a "no slip" condition is applied over the membrane and a "slip" condition is applied on the lateral faces. For the electrical conductivity case, an electrical potential difference is applied between the top and bottom faces, and the electrical field is parallel to all the others faces (membrane and lateral faces).

where $\phi$ is the porosity, $\sigma_{f}$ the electrical conductivity of the fluid filling the pore space, $F$ the formation factor (like $\alpha_{\infty}$, a scale invariant property).

The macroscopic behavior of porous media regarding the transport of viscous fluid and electrical current, results from the cumulative effects of specific local mechanisms. In the case of foam permeability [9], the dominating local mechanism of viscous fluid flow corresponds to the flow passing through a single membrane aperture. Hereafter, we reexamine local mechanisms governing electrical conduction properties in order to elaborate on the simplest description that provides a reliable estimate of the tortuosity $\alpha_{\infty}$ in micro-macro models of partially closed foam.

\section{TWO PORES CONNECTED BY A SINGLE PERFORATED MEMBRANE}

In this section, we consider the simplest model of foam: two pores connected by a thin membrane containing a circular hole of radius $r_{o}$ (Fig. 1). Periodic conditions are applied on the lateral faces of the pore space. By using FEM simulation, we determine the effective fluid flow conductance $G_{f l}$ (defined as the ratio of the fluid flow to the pressure difference applied between the opposite faces of the pore space volume) and the effective electrical conductance $G_{e}$ (defined as the ratio of the total current to the electrical potential difference applied between the opposite faces of the pore space volume). A commercial code, Comsol Multiphysics v5.2, was used ("creeping flow" and "electrostatic" modules). Details of the boundary value problems solved for each fundamental case are given in appendix A.

Figure 2 shows that the fluid flow conductance is

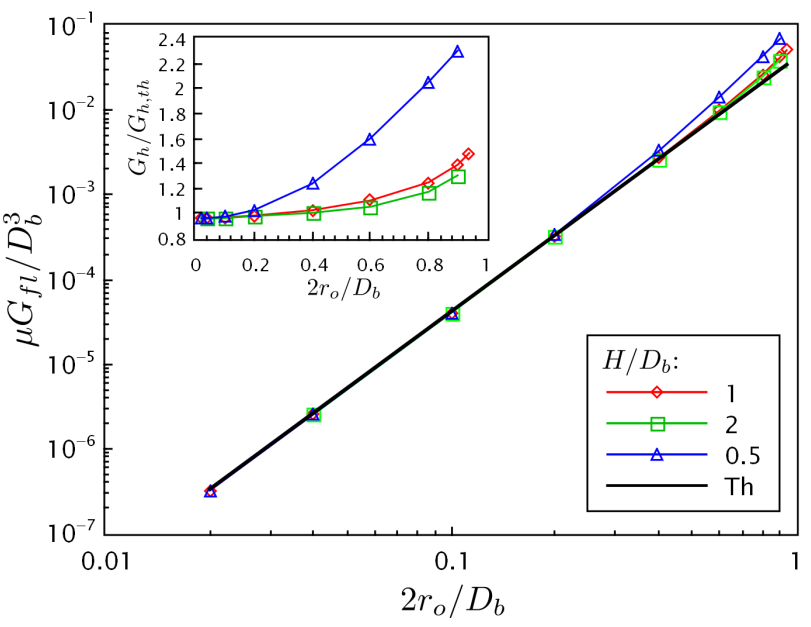

Figure 2. Fluid flow conductance $G_{f l}$ of two inter-connected pores as a function of the membrane aperture size for various aspect ratio $H / D_{b}$. Dots are the FEM results. Full line is plotted by using Sampson's equation.

strongly dependent on the membrane aperture and quite insensitive to the aspect ratio $H / D_{b}$ of the pore space. This result shows that the viscous losses are essentially dominated by a mechanism acting in the vicinity of the membrane aperture. Indeed, when the membrane disappears $\left(2 r_{o} / D_{b} \longrightarrow 1\right)$, the viscous loss disappears and the fluid flow conductance diverges (Fig. 2b). Moreover, as shown in [9], the fluid flow conductance can be estimated by a Sampson law [17]:

$$
G_{f l}=\frac{r_{o}^{3}}{3 \mu}
$$

Figures 3 and 4 show that the electrical conductance increases when the membrane aperture size increases. For low membrane aperture sizes, the electrical conductance exhibits a linear dependence with the aperture size (inset graph of Fig. 3b). Note that, when the membrane thickness is taken into account, this linear dependence is no longer observed at very low membrane aperture sizes. If the electrical conductivity and the permeability follow the same trend as previously described, they have a different behavior when the membrane aperture reaches the lateral side of the pore space. Indeed, unlike permeability, the electrical conductance tends to a finite value equal to $D_{b}^{2} \sigma_{f} / H$ where $\sigma_{f}$ is the electrical conductivity of the fluid. This result is fundamental because it reveals that the whole pore space, and not only a part located in the vicinity of the membrane aperture, contributes to the electrical conductivity.

In the last century, the effect of constrictions on electric conductance (Fig. 5) was studied by several authors coming from different scientific communities. Hall [18] showed that the electrical resistance $R_{e}$ (the inverse of the electrical conductance $G_{e}$ ) due to a circular aperture in an isolating membrane immersed in a conducting fluid 

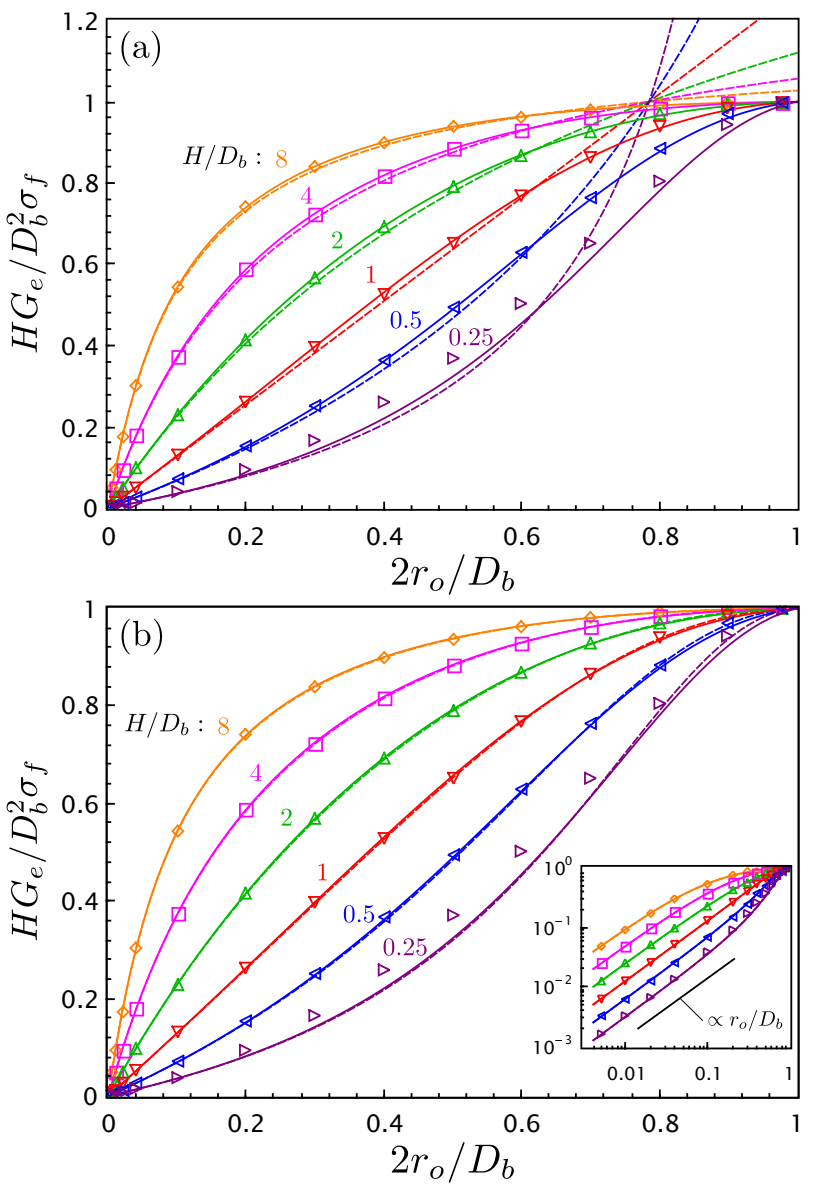

Figure 3. Cylindrical pores: electrical conductance $G_{e}=$ $1 / R_{e}$ of two inter-connected pores normalized by the electrical conductance without membrane $\left(D_{b}^{2} \sigma_{f} / H\right)$ as a function of the membrane aperture size for various aspect ratios $H / D_{b}$. (a) Comparison between FEM results (dots), predictions of Eq. 6 from Sahu \& Zwolak (dashed lines) and Eq. 7 (full lines). (b) Comparison between FEM results (dots), predictions of Eq. 5 from Rosenfeld and Timsit (dashed lines) and Eq. 7 (full lines). The same data are used in graph (b) and in its inset graph.

having an infinite size (electrodes are located at infinity) is given by $1 / R_{e}=2 \sigma_{f} r_{o}$. Earlier, in the context of electrical conduction through two conducting bodies in contact, several authors [20-22] showed that electrical resistance for low area of contact between bodies is given by $1 / R_{e}=2 \sigma_{f} r_{c}$ where $r_{c}$ is the contact radius. Later, Rosenfeld and Timsit [19] found the exact solution for the electrical resistance of a long constricted cylinder (cylinder length $\gg$ constriction radius $=$ membrane aperture radius $): R_{e}=\left(H / \sigma_{f} A_{c}\right)+R_{s}$ where $A_{c}$ is the crosssectional surface of the tube $\left(A_{c}=\pi D_{b}^{2} / 4\right.$ where $D_{b}$ is the cylinder diameter). The first term $H / \sigma_{f} A_{c}$ corresponds to the resistance of the cylinder without constriction, and the second term $R_{s}$ is the spreading resistance due to the constriction effect. As the exact solution found by Rosenfeld and Timsit implies an infinite sum of terms, the authors gave a compact expression for the spreading

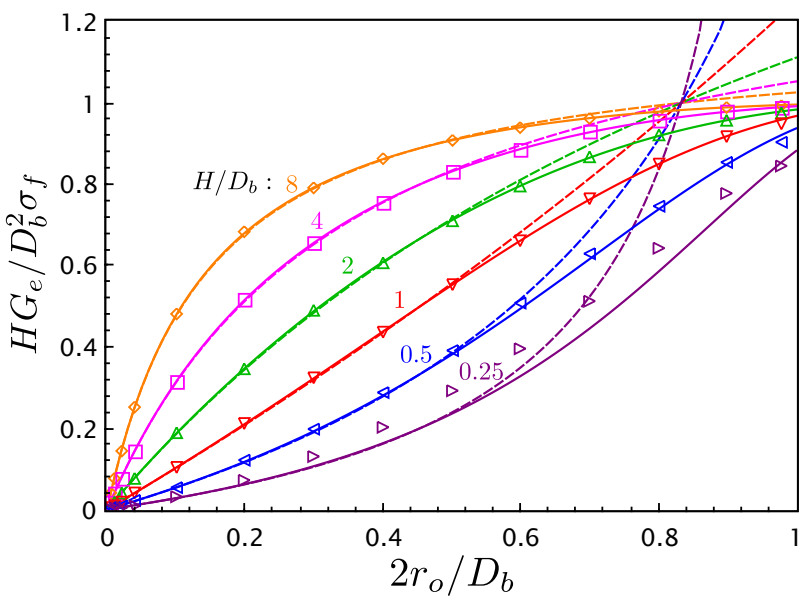

Figure 4. Rectangular pores: normalized electrical conductance $G_{e}=1 / R_{e}$ of two inter-connected pores as a function of the membrane aperture size for various aspect ratios $H / D_{b}$. Dots are the FEM results. Dashed and full lines are plotted by using, respectively, Eq. 6 and Eq. 7.

resistance $R_{s}$. The total resistance is then given by:

$$
R_{e}=\frac{H}{A_{c} \sigma_{f}}+R_{s}=\frac{H}{A_{c} \sigma_{f}}+\frac{1}{2 \sigma_{f} r_{o}} P\left(\frac{r_{0}}{D_{b}}\right)
$$

where $P(x)=\left[1+C_{1} x+C_{2} x^{2}+C_{3} x^{3}+C_{4} x^{4}\right]$ with $C_{1}=-2.83162, C_{2}=0.25288, C_{3}=1.22088, C_{4}=$ 3.19968 .

Note that the polynomial $P$ is built with the constraints, $P(1 / 2)=0$ and $P(0)=1$ with the aim of recovering the asymptotic solutions $\left(r_{o} \rightarrow D_{b} / 2\right.$ corresponds to a finite uniform cylinder and $r_{o} \rightarrow 0$ corresponds to an infinite constricted cylinder).

Recently, Sahu and Zwolak [23] have studied the resistance of two interconnected pores having cylindrical or rectangular shape and finite size with various aspect ratios $H / D_{b}$. The authors break down the pore volume into several parts, each contributing to the electrical conductance of the pore space (Fig. 5). The first subpart, only observed for long cylinders $\left(H>D_{b}\right)$, is located far from the membrane aperture where the current streamlines are almost parallel to the external electrical field as in a uniform conductor. The third subpart is located in the vicinity of the membrane aperture where the current streamlines converge to the aperture. The second subpart is a transition region between the first and the third, where the current streamlines change their behavior. The fourth subpart corresponds to the small channel inside the membrane thickness itself. The first and fourth subparts can be approximated by uniform conductors having a known resistance $\left(R_{I}=\left(H-L_{2}\right) / \sigma_{f} A\right.$ with $L_{2} \approx D_{b} / 2$ and $R_{I V}=h_{m} / \pi \sigma_{f} r_{o}^{2}$ respectively). For the third subpart, Sahu and Zwolak use an approximation based on the Taylor expansion of the analytical solution for the access resistance with a spheroidal 
electrode: $R_{I I I}=\frac{1}{2 \sigma_{f} r_{o}}\left(1-\frac{2 r_{0}}{\pi L_{1}}+O\left(\left[\frac{r_{0}}{L_{1}}\right]^{3}\right)\right)$ where $L_{1} \approx D_{b} / 2$ is the size of the spheroid region (Fig.5). For the transition region, the authors consider a contribution proportional to $R_{I I}=1 / \sigma_{f} D_{b}$. Therefore, by considering a series connection of the sub-part resistances and after some calculations, the total resistance $R_{e}$ is given by:

$$
R_{e}=\frac{H}{\Psi \sigma_{f} D_{b}^{2}}+\left[\frac{1}{\sigma_{f}}\left(\frac{1}{2 r_{o}}-\frac{f_{l}}{\Psi D_{b}}+\frac{h_{m}}{\pi r_{o}^{2}}\right)\right]
$$

where $\Psi$ and $f_{l}$ are coefficients depending on the geometric details of the cell: $\Psi=1$ and $f_{l} \approx 1.2$ for rectangular pores, or $\Psi=\pi / 4$ and $f_{l}=1.0$ for cylindrical pores [24]. Note that the authors determined the coefficients $f_{l}$ by adjusting the equation's predictions to their numerical simulation results.

The term in brackets in Eq. 6 corresponds to the spreading resistance $R_{s}$ as defined by Rosenfeld and Timsit. Similarly to the case of long constricted cylinder, the spreading resistance of a finite constricted cylinder should be equal to zero when the membrane aperture is equal to the cross-section radius or, more precisely, when the surface of the membrane aperture is equal to the cross-section surface $\left(2 r_{o}=D_{b}\right.$ for cylindrical pores, and $\sqrt{\pi} r_{0}=D_{b}$ for rectangular pores). However, the equation given by Sahu and Zwolak do not satisfy this constraint since $f_{l} \neq \Psi$ for cylindrical pores and $D_{b}=H$. Therefore by comparison with FEM results, we show in Fig. 3a that Eq. 6 cannot predict the electric resistance for high membrane aperture size $\left(2 r_{0} \rightarrow D_{b}\right)$.

Because we aim at generating an expression enabling us to recover bulk and membrane effects for the whole range of membrane aperture sizes (and not only for tiny holes corresponding to graphene nanopores) with great accuracy, a modification of Eq. 6 is further proposed in order to improve the transition region mixing the access and bulk like behavior:

$R_{e}=\frac{H}{\Psi \sigma_{f} D_{b}^{2}}+\frac{1}{\sigma_{f}}\left[\frac{1}{2 r_{o}}\left(1+C_{1} \frac{r_{o}}{D_{b}}+C_{3}\left(\frac{r_{o}}{D_{b}}\right)^{3}\right)+\frac{h_{m}}{\pi r_{o}^{2}}\right]$

with $C_{1}=-2.89$ and $C_{3}=-8\left(1+\frac{1}{2} C_{1}\right)$ for cylindrical pores; $C_{1}=-2.52$ and $C_{3}=-\pi^{1.5}\left(1+\frac{1}{\sqrt{\pi}} C_{1}\right)$ for rectangular pores.

Note that $C_{1}$ is determined by fitting the equation's predictions to our numerical simulation results ; and $C_{3}$ is chosen with the aim of recovering the resistance without membrane $R_{e} \sigma_{f}=\frac{H}{\Psi D_{b}^{2}}$ (by neglecting the membrane thickness term). The relative difference between FEM simulations results and resistances calculated by using Eq. 7 does not exceed $1 \%$ as long as the aspect ratios $H / D_{b}$ are superior to 0.75 and the aperture size $r_{o}$ is inferior to $0.9 D_{b}$. Figure 3 a shows that Eq.7 predicts FEM results for a wide range of membrane aperture size with a better accuracy than Eq. 6. Moreover, Figure 3b compares the prediction of Eq. 7 to the solution given by Rosenfeld and Timsit (Eq. 5 ). Both equations have identical values of resistance except when the membrane aperture size is close to the radius of the cylinder. In this case, predictions of Rosenfeld and Timsit equation are slightly better even for short cylinders. However, for very short cylinders $\left(H / D_{b} \leq 0.25\right)$, all equations fail in their predictions. Finally, in Fig. 4, we compare both the predictions given by Sahu and Zwolak (Eq. 6) and provided by Eq. 7 to FEM results on rectangular pores. As observed for the case of cylindrical pores, Eq. 7 predicts FEM results for a wide range of membrane aperture size with a better accuracy than the Sahu and Zwolak expression.

Before moving to the next section, we briefly discuss the golden aspect ratio introduced by Sahu and Zwolak $[23,24]$. For this special aspect ratio $\left(H / D_{b}\right)^{*}$, finite size effects should be eliminated in numerical simulations (i.e. for any $L$ or $D_{b}$ such as $H / D_{b}=\left(H / D_{b}\right)^{*}$, $\left.R_{e}=\lim _{\left\{L / D_{b}\right\} \rightarrow \infty}\left(R_{e}\right)\right)$. According to Eqs. 5 or 7 , it is possible to find a particular aspect ratio for which the first term of the spreading resistance expansion, $\frac{1}{2 r_{o}} C_{1} \frac{r_{o}}{D_{b}}$, and the bulk resistance $\frac{H}{\Psi D_{b}^{2}}$ cancel each other out. The golden aspect ratio is then given by $\left(H / D_{b}\right)^{*}=-C_{1} \Psi / 2$, and is equal to 1.26 for rectangular pores, and 1.13 for cylindrical pores (or 1.11 if the Rosenfeld and Timsit equation is used for the spreading resistance). As these values of golden aspect ratios are in the range of aspect ratios where Eqs. 5 and 7 give accurate predictions, our calculation is justified. Note that Sahu and Zwolak [23] find 1.2 for rectangular pores, and 1.07 for cylindrical pores from FEM simulations and molecular dynamics simulations. Finally, finite effects are cancelled as long as the higher terms in the expansion of the spreading resistance are negligible compared to one. From Rosenfeld and Timsit equation's (Eq. 5), this condition is verified with a relative error inferior to $1 \%$ if $2 r_{o}<0.28 D_{b}$.

\section{ORDERED KELVIN FOAM}

In this section we describe the properties of high porosity ordered foam exemplified by a Kelvin structure. As shown in Fig. 6, a periodic unit cell (PUC) of characteristic size $D_{b}$ is used to represent the pore structure in foam samples [10, 25]. The number of pores, $N_{p}$, contained within the unit cell is equal to 2 . The cell is based on the Kelvin partition and is a 14-sided polyhedron (8 hexagonal and 6 square faces) corresponding to windows shared with the $N_{v}=14$ neighbors. The cell skeleton is made of idealized ligaments having length $L=D_{b} /(2 \sqrt{2})$ and an equilateral triangular cross section of edge length $r=0.58 D_{b}(1-\phi)^{0.521}$, where $\phi$ is the gas volume fraction. The thickness of the membranes is taken equal to $h_{m} / L=0.001$, such that the effect of the channel inside the membrane thickness can be neglected (because 


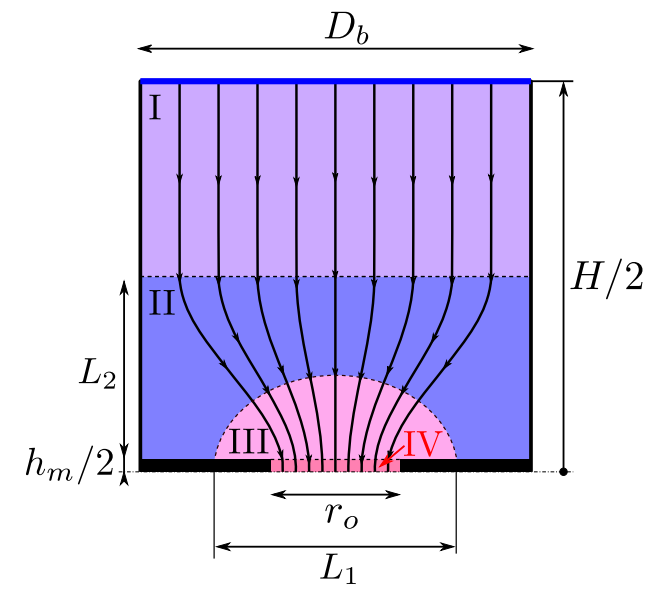

Figure 5. Shape of current streamlines in a pore associated to four regions: I. bulk like, II. transition region, III. access region and IV. membrane thickness channel. Note that the first region is observed only if the pore is long enough $(H \gtrsim$ $\left.D_{b}\right)$.

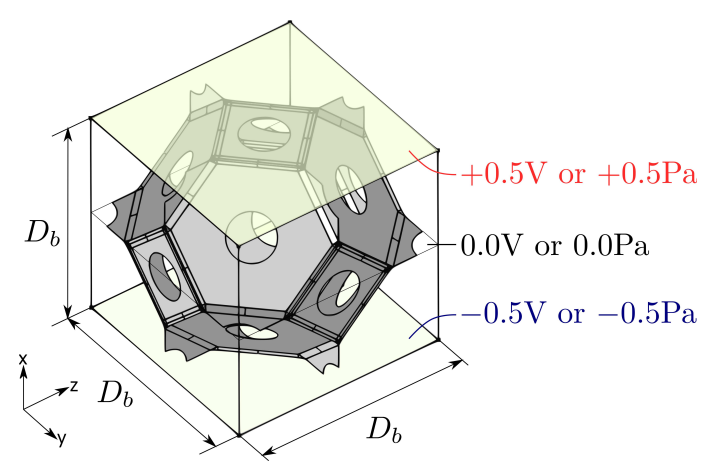

Figure 6. Periodic unit cell based on the Kelvin structure $(\phi=0.995)$. Depending on the physical problem considered, the boundary conditions are different.

$\left.h_{m} \ll r_{o}\right)$. As we are interested in the effect of partial closure of the cell windows by membranes, we partially close the windows by adding holed membranes having a circular aperture of radius $r_{o}$. Note that, in the reference configuration $K_{0}$ (Figs. 6 and 7a), the 14 windows are fully open (i.e. contain no membrane). However, we study how closed membranes modify the electrical conductivity by testing PUC configurations containing different repartitions of closed membranes as shown on Fig. 7.

In perfect agreement with the previous results of ref. [9], Figure 8 shows that the permeabilities of the simulated PUCs follow the power-law given in Sec. III (Eq.4). Moreover, because most of the pressure drop is located in the vicinity of the membrane aperture, a simplified calculation of the permeability based on the pore-network hypothesis is possible. In this framework, a value of fluid pressure is associated with each pore and the flow between two connected pores is based on the Sampson fluid flow conductance (Fig. 9a\&b). The foam permeabil- (a)
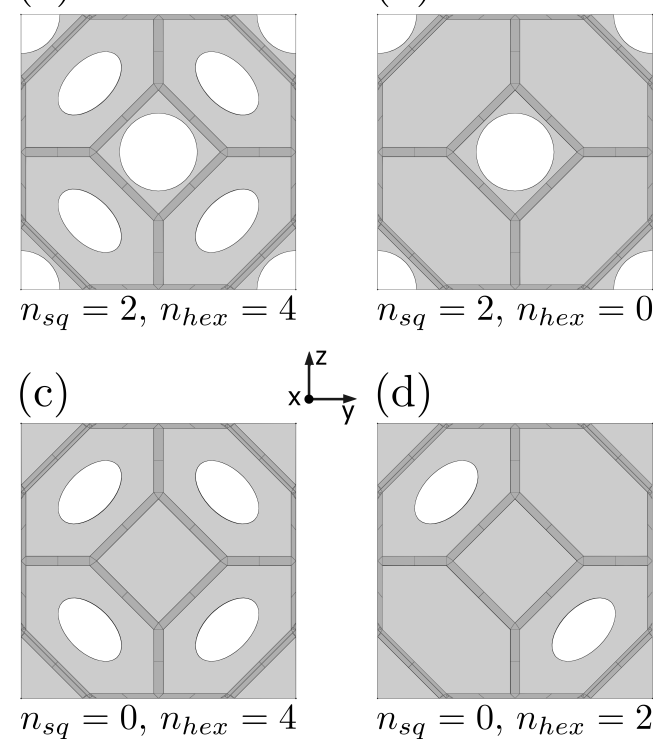

(b)

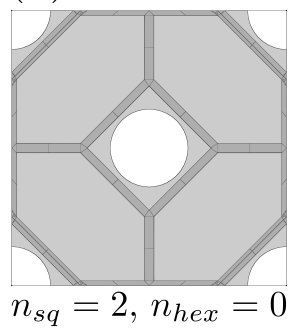

(d)
Figure 7. Top view from the median plane of the tested configurations showing the positions of the closed membranes. Note that the horizontal median plane is a plane symmetry.

ity calculation based on a pore-network model needs to compute the amount of flow passing through the median plane of the PUC, as shown in Fig. 10. Due to the symmetries of the PUC configurations, the pressure difference associated to the flow passing through the square windows is twice the one associated to the hexagonal windows. Therefore it is straightforward to show that the permeability of the studied PUC is given by:

$$
K=\frac{\mu}{D_{b}}\left[n_{s q} G_{f l, s q}+\frac{1}{2} n_{h e x} G_{f l, h e x}\right]
$$

where $G_{f l, s q}$ and $G_{f l \text {,hex }}$ are the fluid flow conductance associated with each window; $n_{s q}$ and $n_{h e x}$ are the numbers of open square and hexagonal windows. Note that from the FEM simulations, $G_{f l, s q}=G_{f l, h e x}=\frac{r_{o}^{3}}{3 \mu}$.

It can be checked that this formula has the ability to predict the whole FEM results as shown by Fig. 8, whichever way the membranes are distributed throughout the PUC.

We further focus on the calculation of foam electrical conduction. As has been shown for the case of two interconnected pores, we also demonstrate here that the electrical conductivity increases with the membrane aperture size and the degree of interconnections between pores (see Fig. 11). For small membrane apertures, the electrical conductivity exhibits a linear dependence on the aperture size. As noticed for the case of two interconnected pores, this behavior also reveals that the membrane aperture governs most of the electrical resistivity for small apertures. In that case, the electrical conduction is dominated by a mechanism acting at the scale of the membrane aperture just as observed for the permeability. Based on this 


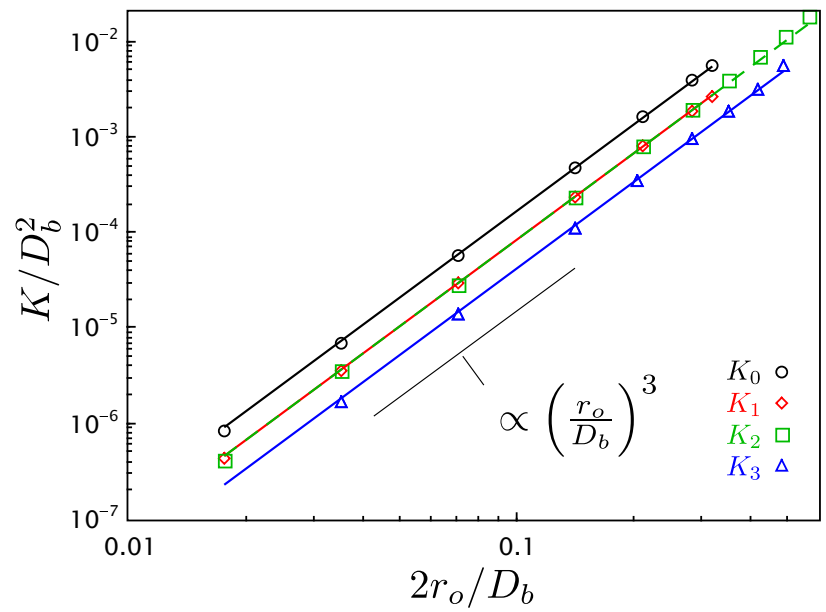

Figure 8. Permeability $K$ of PUC having different configurations of closed membranes for various membrane apertures. Dots are the FEM results. Full and dashed lines are plotted by using Eq.8.

cross-property relation, it seems therefore possible to employ the structure of Eq. 8 in order to calculate the electrical conductivity $\sigma_{e}$. As a result, $K$ is replaced by $\sigma_{e}$, $\mu$ by 1 and $G_{f l}$ by $G_{e}=\sigma_{f}\left[\frac{1}{2 r_{o}}+\frac{h_{m}}{\pi r_{o}^{2}}\right]^{-1}$. Fig. 11a shows that this approach allows us to estimate the electrical conductivity for small aperture, $2 r_{o} / D_{b}<0.1$. By contrast, for higher membrane aperture sizes, the contribution of the pore volume is no longer negligible.

We now focus on the elaboration of a pore-network model able to describe most of the electrical conduction effects in foam. As the whole pore volume must be considered in the electrical conduction issue, the general scheme used for permeability, which only accounts for inter-pore conductances (Fig. 9b), does not apply in this case. Additional intra-pore conductances must be introduced in the network as shown in Fig. 9c. Compared to the pore-network model for permeability which uses only one node for each cell, we introduce 14 nodes per cell in the pore-network model for electrical conduction. The nodes are associated with the different windows of the cell, and are linked to the neighbor windows' nodes sharing the same cell by the intra-pore conductance, and to a node of a neighbor cell by the inter-pore conductance if the membrane separating the cells is opened. Moreover, the neighborhoods of windows are not equivalent: the hexagonal windows within a cell have two kinds of neighbor windows, squares and hexagons, whereas the square windows have only hexagons as possible neighbors. Consequently, we introduce two intra-pore conductances: $G_{i, s h}$ which links square to hexagon, and $G_{i, h h}$ which links hexagon to hexagon. For the inter-pore conductance $G_{e p}$, we consider the term due to the membrane aperture in Eq. 7 with appropriate values of $C_{1}$ and $C_{3}$ : $\frac{1}{G_{e p}}=\frac{1}{2 \sigma_{f} r_{o}}\left(1+C_{1} \frac{r_{o}}{d_{i}}+C_{3}\left(\frac{r_{o}}{d_{i}}\right)^{3}\right)$ where $d_{i}=1 / \sqrt{2 \pi}$ a
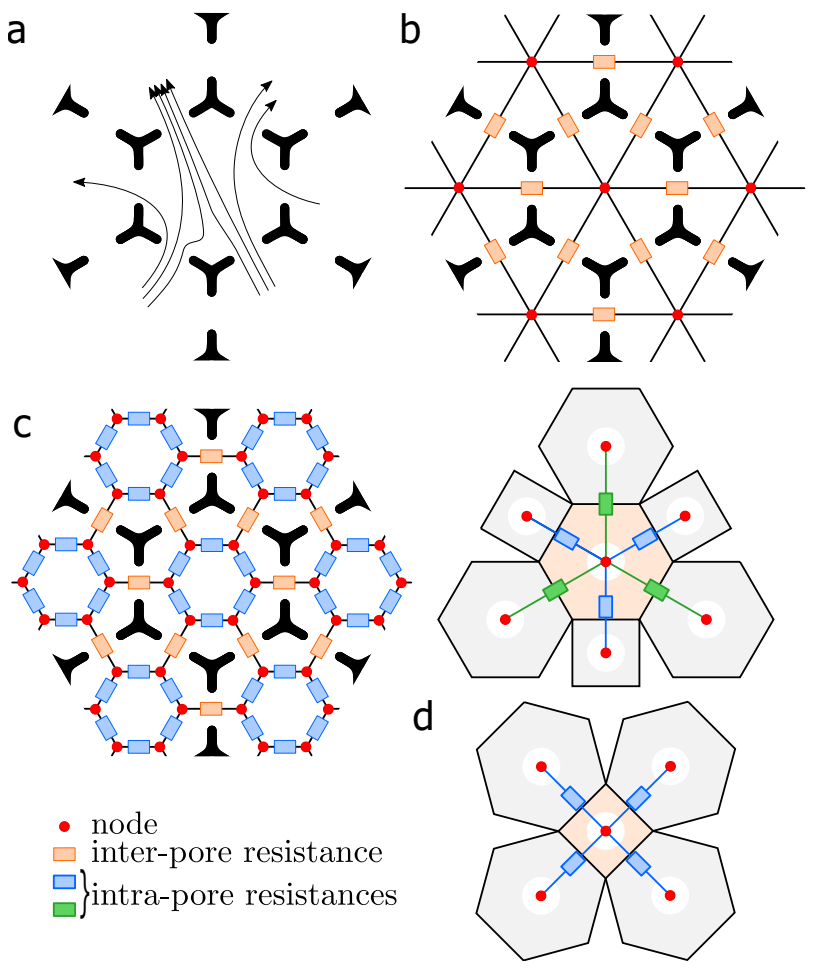

Figure 9. (a) Flow pattern and (b) its equivalent pore-network scheme. (c) Pore-network scheme to solve the electrical conduction problem by using two kinds of conductances: interpore conductance and intra-pore conductances. (d) Intra-pore conductances for square and hexagonal windows of a Kelvin cell.

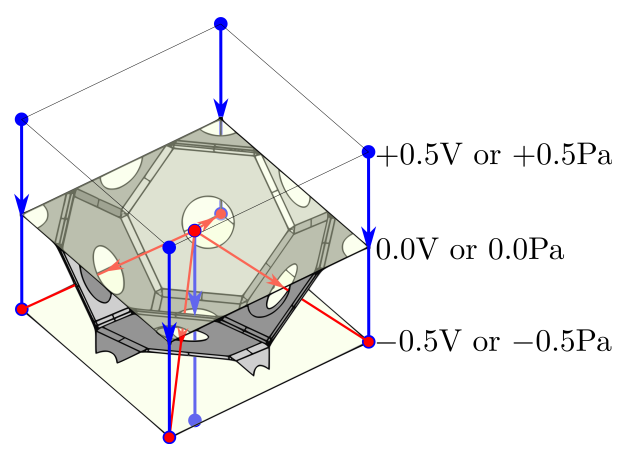

Figure 10. Fluid flow passing through the $K_{0}$ configuration of the PUC (Fig. 7a). Color lines correspond to the active bond in the pore-network model for foam permeability (Fig. $9 \mathrm{~b})$.

for square windows and $\left(3^{1.5} / 4 \pi\right)^{0.5}$ for hexagonal windows. We do not take into account the term involving the thickness of membrane as its effect is negligible in the range of membrane aperture sizes and thicknesses that we investigate $\left(r_{o} \gg h_{m}\right)$. The coefficients, $C_{1}$ and $C_{3}$, and the intra-pore conductances are chosen to best fit the results of FEM simulations. As shown in Fig. 11a, a good agreement between both numerical methods is achieved with: $G_{i, s h} / D_{b} \sigma_{f}=0.16, G_{i, h h} / D_{b} \sigma_{f}=0.30$, 
$C_{1}=-2.53$ and $C_{3}=1.32$. Finally, from a practical point of view, it is possible to estimate the electrical conductivity for the reference configuration $K_{0}$ with a good accuracy (error $<2 \%$ with $\phi=0.995$ ) by using the following equation:

$\sigma_{e, K_{0}}=4 \sigma_{f}\left[\frac{D_{b}}{2 r_{o}}+\frac{h_{m} D_{b}}{\pi r_{o}^{2}}\right]^{-1}\left[1+2.7 \frac{r_{o}}{D_{b}}+5.1\left(\frac{r_{o}}{D_{b}}\right)^{2}\right]^{-1}$

Fig. 11b shows the tortuosities of each PUC configuration for various membrane aperture sizes. When the membrane aperture size is high, the tortuosity is closed to 1 . However, when the membrane aperture size or the number of open windows decreases, the tortuosity increases. Tortuosity can be significantly larger than one in foam containing membranes with small aperture. Therefore this model predicts a significant increase of the dynamic effective density of the effective fluid in the high frequency inertial regime. To reach the inertial regime, the frequency has to be higher than the viscoinertial characteristic frequency $f_{v}$. Fig. 12 shows the visco-inertial characteristic frequency $f_{v}$ as a function of the membrane aperture size for each configuration. For a fully open foam $K_{0}$, the membrane aperture size for which the tortuosity is higher than 10 is equal to $r_{o} / D_{b} \approx 0.0125$. For foam having millimetric pores and filled with air, the corresponding visco-inertial characteristic frequency $f_{v}$ is approximately equal to $100 \mathrm{kHz}$ which is higher than the audible frequency range. However, for foam containing a fraction of closed membrane as in the configuration $K_{3}$ and with $D_{b} \approx 2 \mathrm{~mm}$, the visco-inertial characteristic frequency can reach the audible frequency range $\left(f_{v} \approx 5000 \mathrm{~Hz}\right)$.

As the porosity is a parameter which is involved in the relationship between the tortuosity and the electrical conductivity, we evaluated the effect of porosity on each parameter by performing FEM simulations on K0 configurations for various values of $\phi$. As shown on Fig. 13 , the dependence of the electrical conductivity on $\phi$ is low when compared to the dependence of the tortuosity on $\phi$. This is due to the fact that the effective electrical conductivity $\sigma_{e}$ is only depending on $r_{0}$ when $r_{0} \rightarrow 0$ (bulk effects are vanishing, Eq. 6) whereas the tortuosity involves a factor $\phi$ to account for the scaling from the fluid phase to the overall volume of the porous aggregate (Eq. 3).

\section{ELECTRICAL CONDUCTION IN FOAM WITH RANDOMLY DISTRIBUTED CLOSED MEMBRANES}

Effects of pore network features on electrical conductivity are studied on lattices having a structure similar to Kelvin's structure: each pore is surrounded by $N_{v}=14$ neighbor pores. The samples have a size $L^{2} H_{s p}=16^{3}$ ( $D_{b}$ units) and contain 8192 pores. As we are interested
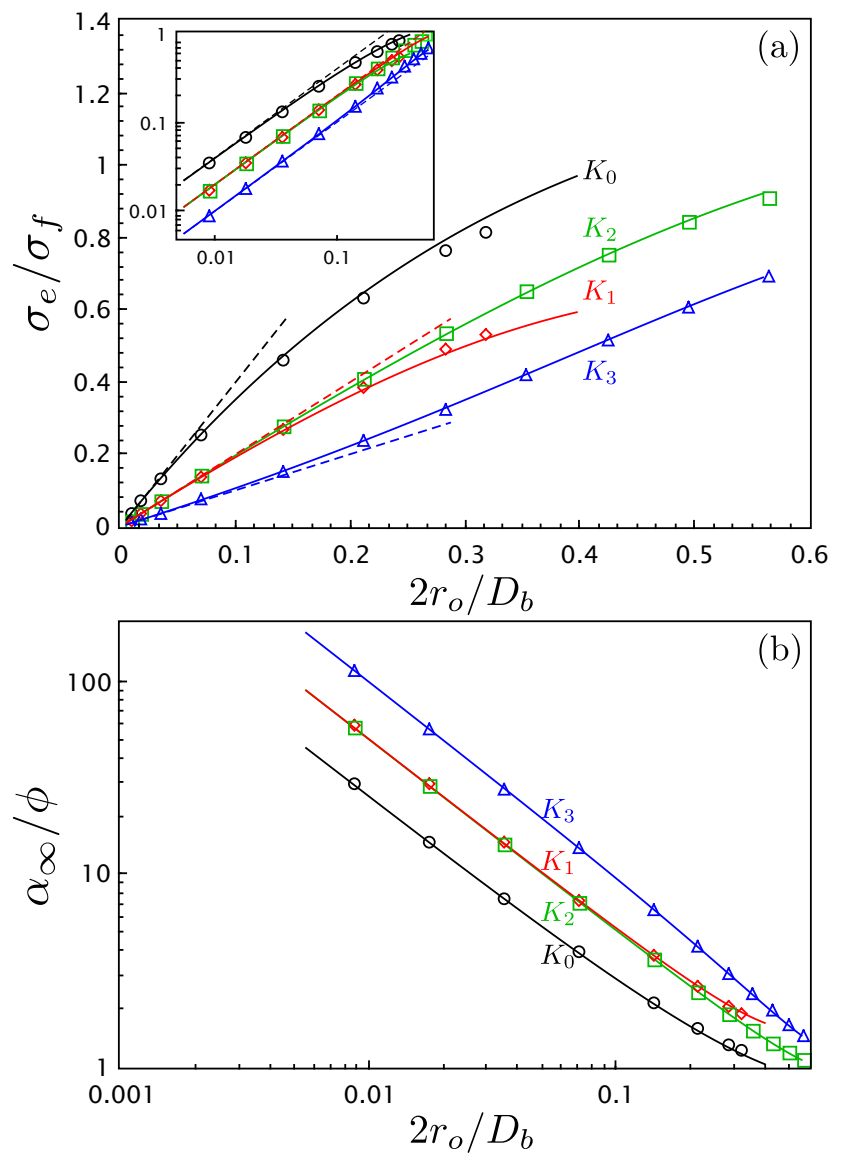

Figure 11. (a) Electrical conductivity $\sigma_{e}$ and (b) tortuosity $\alpha_{\infty}$ of the PUCs as a function of the membrane aperture size for various configurations of closed membranes $\left(K_{0}, K_{1}, K_{2}\right.$, $K_{3}$ ) with $\phi=0.995$. The same data are used to the inset graph of (a). Dots correspond to the FEM results. Dashed lines are plotted by using Eq. 8 taking into account only interpore electrical conductances (Fig. 9b). Full lines are plotted by using pore-network calculations taking into account both intra- and inter-pore electrical conductances (Fig. 9c). Due to the symmetries of the PUC configurations, analytical expressions for effective conductivity can be found for each configuration (see appendix B).

in the effect of closed membranes on electrical conduction, we close a part of the windows by randomly cancelling some electrical bonds between two nodes connecting two pores. The values of intra-pore conductances, $G_{i, s h}$ and $G_{i, h h}$, found previously on Kelvin foam having $\phi=0.995$ are used in this pore-network calculation. Boundary effects are avoided by resorting to periodic conditions imposed in the directions perpendicular to the macroscopic gradient. Details of the calculations of electrical conductivity are given in appendix B.

Fig. 14 shows the electrical conductivity calculated with simulations performed on large samples having random positions of closed windows and various open window fractions $x_{\text {ow }}$ having identical membrane aperture sizes. Below a critical concentration $x_{o w}<x_{p} \approx 0.1 \approx$ 

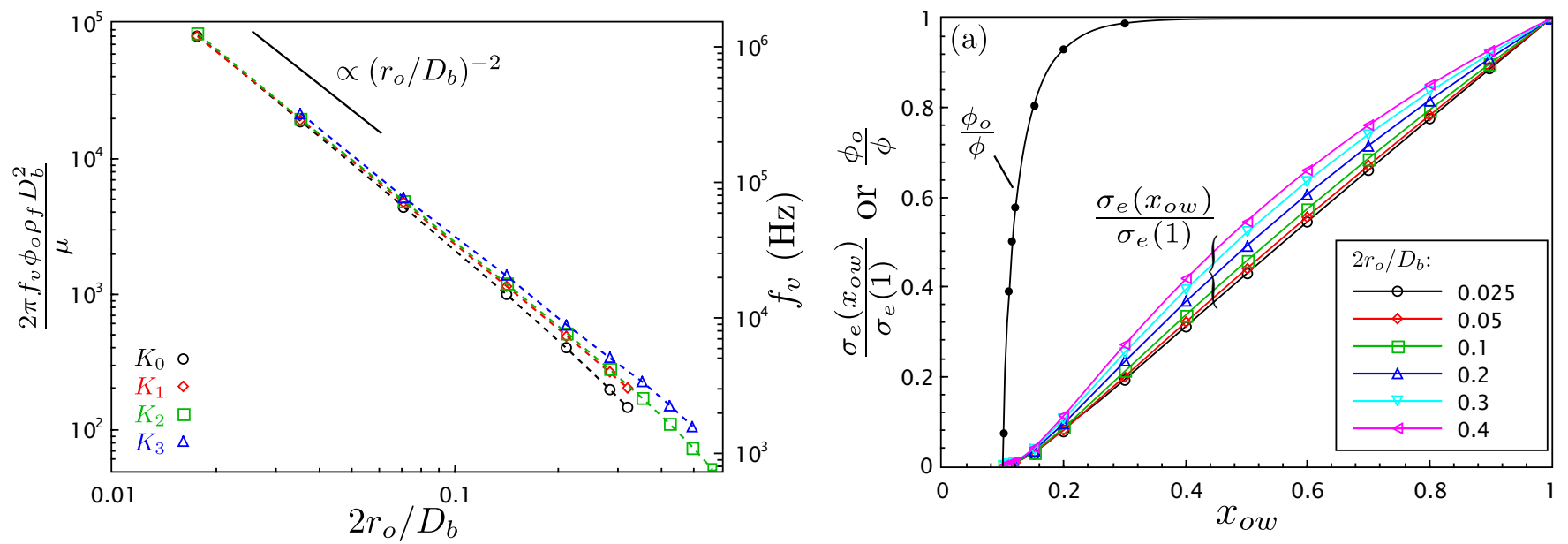

Figure 12. Visco-inertial characteristic frequency $f_{v}$ of $K_{i}$ configuration as a function of the aperture size calculated from FEM results: on the left abscissa, the frequency is normalized, and on the right abscissa, the frequency corresponds to the case of foam filled by air with $D_{b}=1 \mathrm{~mm}$ and $\phi \approx 1$.
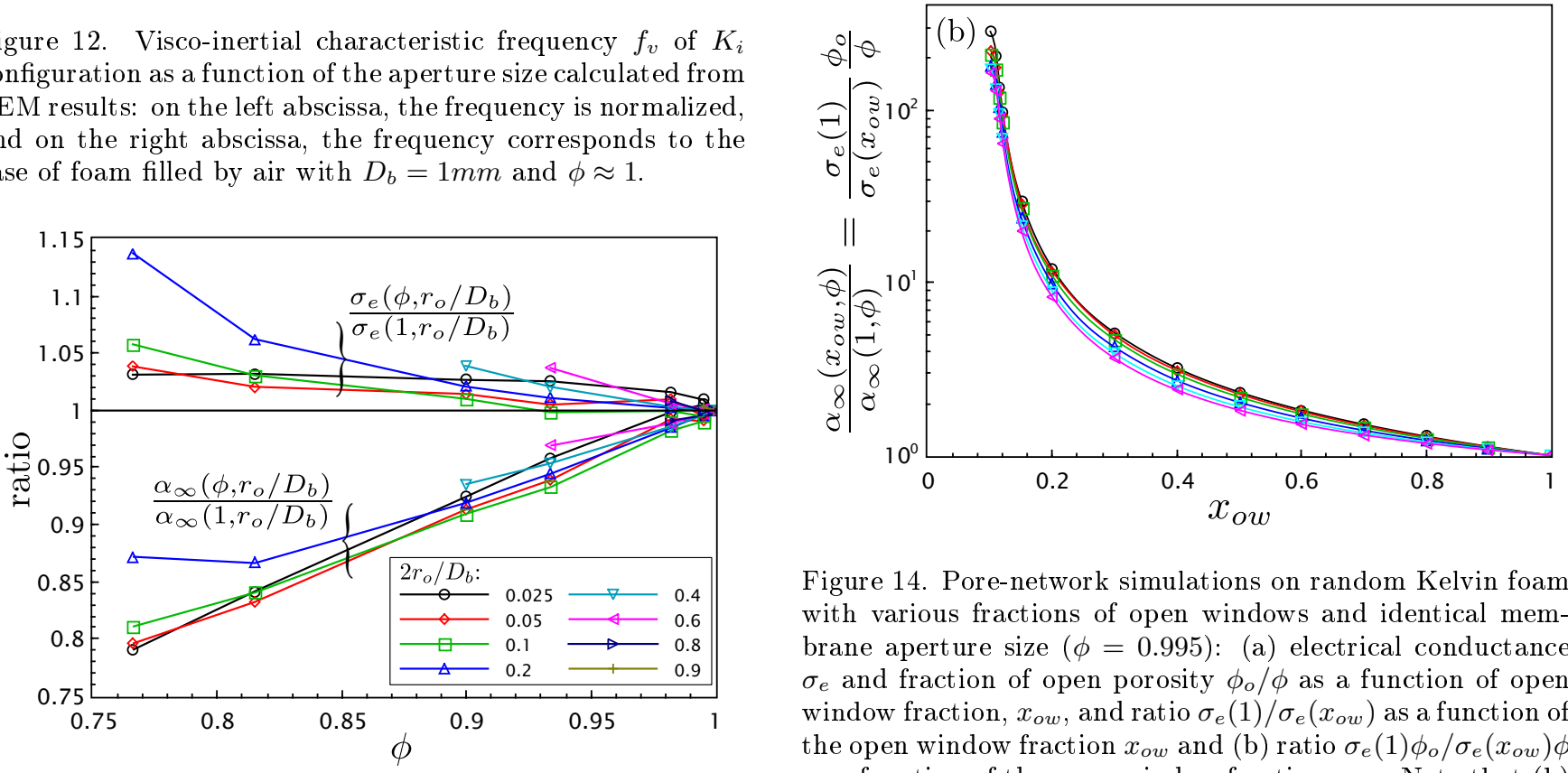

Figure 14. Pore-network simulations on random Kelvin foam with various fractions of open windows and identical membrane aperture size $(\phi=0.995)$ : (a) electrical conductance $\sigma_{e}$ and fraction of open porosity $\phi_{o} / \phi$ as a function of open window fraction, $x_{o w}$, and ratio $\sigma_{e}(1) / \sigma_{e}\left(x_{o w}\right)$ as a function of the open window fraction $x_{o w}$ and (b) ratio $\sigma_{e}(1) \phi_{o} / \sigma_{e}\left(x_{o w}\right) \phi$ as a function of the open window fraction $x_{o w}$. Note that (b) leads to the ratio $\alpha_{\infty}\left(x_{o w}\right) / \alpha_{\infty}(1)$ in considering for the pore space volume to use in the definition of the tortuosity, the open pore space. Moreover, $\sigma_{e}(1)=\sigma_{e, K_{0}}$ can be calculated by using Eq.9.

$1.5 / N_{v}$, the network of interconnected pores does not connect the top of the sample to the bottom. Therefore the open porosity (porosity of the percolating pore space) is null and no electrical conduction through the foam is possible. In the range $[0.1,0.2]$ of open window fraction, on the one hand the fraction of open porosity increases from 0 to 0.93 , and on the other hand, the electrical conductance gently rises. For $x_{\text {ow }}>0.2$, the electrical conduction gradually increases as the fraction of open windows rises. For low aperture size, the electrical conduction exhibits a quasi-affine dependence on the open window fraction $x_{\text {ow }}$ similar to the one observed in [9] for permeability. As we previously saw, the fluid flow conductance corresponds to an inter-pore conductance, Therefore as the inter-pore resistance dominates the electrical conduction at low membrane apertures, a similar behavior between electric conductivity and per-

meability was expected for low membrane apertures. In that case, electrical conductivity and tortuosity can be estimated by using the effective model for foam permeability of Langlois et al. [9] (see appendix C).

\section{CONCLUSION}

In order to study the effects of both the fraction of open windows and their aperture sizes on both electric conductivity and high frequency tortuosity of foam, we performed simulations at different scales: 1) FEM simulations computing the flow of electric current at the pore scale and on ordered Kelvin foam; and at the higher scale, 2) pore-network simulations capturing the physics 
at the lower scale without describing the entire velocity field. Effects of the membrane aperture size and the open window fraction on the permeability are recalled to highlight the difference between permeability and electrical conductivity. The FEM simulations at pore scale were useful to identify the mechanisms of electric conduction through a single pore. The analysis reported in this paper shows that, for low membrane aperture size, the foam electrical conductivity can be described by a mechanism acting at the scale of the membrane aperture. However, when the membrane aperture size is comparable to the pore size, another mechanism acting on the whole pore volume must be considered. By considering these results, we build a pore-network model at the pore scale by using two kinds of conductance: inter-pore conductance and intra-pore conductance. By using appropriate conductances, we show that pore-network model can reproduce the FEM results performed on ordered Kelvin foam having different configurations of closed membranes. Pore-network simulations on large samples having random position of closed windows show that percolation occurs when the fraction of open windows is close to $x_{p}=1.5 / N_{v}\left(\approx 0.11\right.$ for Kelvin foam having $\left.N_{v}=14\right)$ and that the fraction of closed windows can significantly increase high frequency tortuosity.

An experimental confirmation of this numerical study would require the production of well-controlled monodisperse foam having a known (controlled or measured) fraction of closed membranes. Emulsion and foam templating techniques can be used to produce tailor-made porous polymers, see for instance Ref.[26] and references therein. In particular, experimental results were reported [27] to generate porous polyurethane by a sphere templating method as an attempt to control the interconnection size for a given bead diameter (bead sintering). We are not aware of any experimental setup able to produce well-defined (geometrically) polymeric structures having closed and open pores of different ratios. Millifluidic techniques were, however, employed to generate monodisperse foams with various membrane contents [10]; (geometrically) characterized according to the proportions of closed and open windows and aperture size. While tortuosity estimates were reported using acoustical measurements [28] by Trinh et al. [10], the acoustical method failed to provide tortuosity results when the membrane content was too high. This suggests that the acoustical method is not appropriate for the characterization of foam having a large proportion of fully closed membranes and/or very low membrane aperture sizes due to the low amplitude of signal transmitted through the sample at the third microphone and the high values of the viscoinertial frequency (i.e. the inertial regime is out of the audible frequency range). Moreover, in all treatments of acoustical characterization it is assumed that the fluid motion is completely decoupled from the solid motion. This is certainly not true if the tortuosity is much larger than one. For example, in the case of porous media with soft and/or lightweight frame, sound propagation through fluid can induce frame resonance at specific frequencies as observed in [8]. It should be possible in the future to see a comparison between the predictions of the current model and the results from direct experiments using alternative techniques, such as (i) electrical conductivity measurements [4, 29], (ii) superfluid acoustics (He II) taking advantage of the fact that the acoustics of He II can become identical with the acoustics of an ideal fluid [29, 30], or (iii) ultrasonic measurements of velocity dispersion in porous media saturated by various fluids [31].

We conclude this section with a summary of this main results:

(1) A unified set of viscous fluid flow and electrical conduction calculations have been carried out on a family of idealized three-dimensional model foams with emphasis on membrane effects.

(2) We first studied ordered cellular foams, for which excellent analytical estimates of the permeability $K$ and tortuosity $\alpha_{\infty}$ were provided. More particularly, the structure of the equations predicting $K$ and $\alpha_{\infty}$ is determined based on phenomenological considerations and the relevant coefficients fitted against numerical results. Moreover, the radius of a perforated membrane $r_{0}$ is the most important geometrical parameter governing the viscous fluid flow and electrical conduction properties. This is especially true when $r_{0} / D_{b} \rightarrow 0$.

(3) Estimates of $\alpha_{\infty}$ were derived based on a bulk and a membrane effect contribution, the later mechanisms dominating the overall effective properties of the flow of the electric current when $r_{0} / D_{b} \rightarrow 0$.

(4) The relation $K / D_{b}^{2} \propto\left(r_{0} / D_{b}\right)^{3}$ based on the Sampson law appears to be valid over the entire range of membrane aperture with different configurations of closed membranes in Kelvin cells.

(5) These analytical results then enabled the use of pore-network simulations to study the behavior of the tortuosity, including percolation effects in foams containing large number of cells $\left(\sim 16^{3}\right)$ having randomly distributed closed membranes. It is quite interesting to see that this leads to a drastic increase of the tortuosity $\alpha_{\infty}$ with the closed window fraction, $1-x_{\text {ow }}$.

(6) Finally, we examine the relationship between permeability $K$ and tortuosity $\alpha_{\infty}$ when both the aperture size $r_{0} / D_{b}$ and the membrane thickness $h_{m}$ are very small: $K / D_{b}^{2} \propto\left(r_{0} / D_{b}\right)^{3}$ and $\alpha_{\infty} \propto \phi /\left(r_{0} / D_{b}\right)$.

\section{Appendix A: calculation of transport parameters}

In this section, we briefly introduce the boundary value problem used for computing the permeability and the electric conductivity.

Viscous flow: The low Reynolds number flow of an incompressible Newtonian fluid is governed by the usual Stokes equations in the fluid phase [32]:

$$
\eta \mathbf{\Delta} \mathbf{v}-\nabla p=-\nabla p^{m} \text { with } \nabla \cdot \mathbf{v}=0 \text { in } \Omega_{f}
$$




$$
\begin{gathered}
\mathbf{v}=0 \text { on } \partial \Omega \\
\mathbf{v} \text { and } p \text { are } \Omega-\text { periodic }
\end{gathered}
$$

where $\nabla p^{m}$ is the macroscopic pressure gradient acting as a source term. Symbols $\mathbf{v}$ and $p$ are the velocity and pressure of the fluid, respectively.

It can be shown that the local field of the static viscous permeability is obtained from the local velocity field as $\mathbf{v}=-\mathbf{k}_{0} / \eta \nabla p^{m}$.

The static viscous permeability $k_{0}$ is calculated by the standard definitions below,

$$
k_{0}=\phi\left\langle\mathbf{k}_{0}\right\rangle \text {, }
$$

Inertial flow: At the high frequency range with $\omega$ large enough, the viscous boundary layer becomes negligible and the fluid tends to behave as perfect, since it has no viscosity. Consequently, the perfect incompressible fluid formally behaves according to the electrical conduction problem [33]:

$$
\begin{gathered}
\nabla . \mathbf{E}=0 \text { with } \mathbf{E}=-\nabla \varphi+\mathbf{e}, \quad \text { in } \Omega_{f} \\
\mathbf{E . n}=0, \quad \text { in } \partial \Omega_{f} \\
\varphi \text { is } \Omega-\text { periodic }
\end{gathered}
$$

where $\mathbf{e}$ is a given macroscopic electric field, $\mathbf{E}$ the solution of the boundary problem having $-\nabla \varphi$ as a fluctuating part, and $\mathbf{n}$ is unit normal to the boundary of the pore region.

The high frequency tortuosity $\alpha_{\infty}$ is calculated,

$$
\alpha_{\infty}=\frac{\left\langle\mathbf{E}^{2}\right\rangle}{\langle\mathbf{E}\rangle^{2}}
$$

\section{Appendix B: Pore-network simulations}

The general principle of calculation of the electric conductivity of a tube network as a model of porous media can be found in Johnson et al 1987. Details about the use of pore-network simulation for the calculation of foam permeability, can be found in [9]. Hereafter, we give some details about the pore-network simulations for the case of foam electrical conduction.

In the pore-network model, the details of electrical potential fluctuations within a single pore are not described in detail. To take into account the current displacement within a pore, the inner volume of a pore is decomposed into 14 parts which are each associated with a node in the electrical pore-network. The nodes are linked by intrapore conductance for two neighbor nodes located within the same pore or by inter-pore conductance for two pores located in two different pores linked by an open window. The first step is to build the conductance network.

We consider, for each node, a unique value of potential without calculating its fluctuations inside the pore. At the local scale, the current $J_{j \rightarrow i}$ from node $j$ to node $i$ is governed by the differential electrical potential difference between the nodes $\Delta V_{i j}=V_{j}-V_{i}$ :

$$
J_{j \rightarrow i}=G_{i j} \Delta V_{i j}
$$

where the coefficient $G_{i j}$ is the conductance between the nodes $i$ and $j$.

At steady state, the sum of current coming from neighbor nodes is equal to zero, leading to: $\sum_{j=1}^{N_{v}} G_{i j}\left(V_{j}-V_{i}\right)=0$. To generate a current displacement through the sample, a potential difference is imposed between the top and bottom faces of the sample $\left(V_{t o p}=\Delta V_{s p}, V_{b o t}=0\right)$. By considering these boundary conditions, the previous equation can take a matrix form:

$$
\mathbf{G V}_{i}=\mathbf{S}_{i}
$$

where $\mathbf{V}_{i}$ is a vector containing the electrical potential of nodes (except that the nodes located on top and bottom faces are excluded); $\mathbf{G}$ is the matrix defined from local conductances $\left(-\sum_{j} G_{i j}\right.$ along diagonal and $G_{i j}$ elsewhere) and $\mathbf{S}_{i}$ is a vector containing zeros except for nodes located on the top face of the sample as neighbors where $S_{i}=-\sum_{j_{\text {top }}} G_{i j_{\text {top }}} \Delta V_{s p}$.

When the pore network is linked from top to bottom of the sample, and by considering only the nodes located in the interconnected pores, $\bar{G}$ can be inverted and the electrical potential in each node can be calculated from Eq.B1. Finally, the macroscopic current $I$ and the effective conductivity $\sigma_{e}$ can be calculated as follows:

$$
\begin{array}{r}
I=\sum_{i_{b o t}} \sum_{j_{v}} J_{j_{v} \rightarrow i_{b o t}}=\sum_{i_{b o t}, j_{v i}} G_{i_{b o t} j_{v i}} \Delta V_{i_{b o t}, j_{v i}} \\
\sigma_{e}=I H_{s p} / L^{2} \Delta V_{s p}
\end{array}
$$

For simple networks, it is possible to derive analytical expressions for the effective electrical conductivity. Due to the symmetries of the PUC configurations (Fig. 7), their equivalent conductance pore-networks can be simplified as shown in Fig. 15. From these equivalent conductance pore-networks, a calculation of the equivalent conductance can be performed leading to the effective electrical conductivity given in Tab. I.

\section{Appendix C: Effective medium model for electric conductance}

Here we present an effective medium model for electric conductivity in the case of small membrane aperture size $\left(2 r_{0} / D_{b}<0.1\right)$. This model is based on the effective medium model of foam permeability detailed in [9]. 

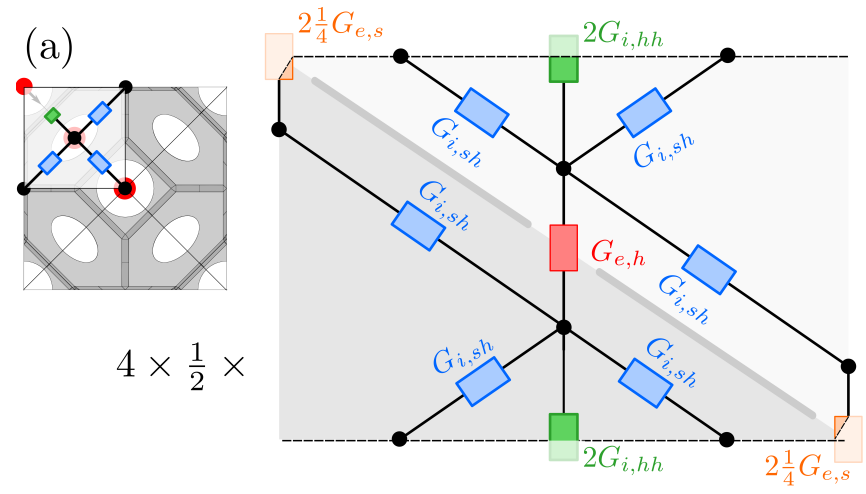

(b)

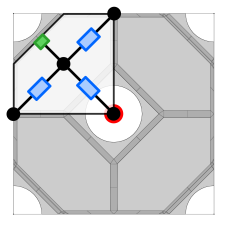

$4 \times 2 \times \frac{1}{4} \times$

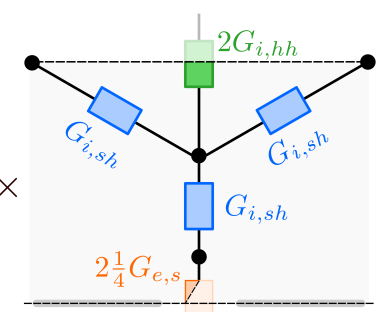

(c)

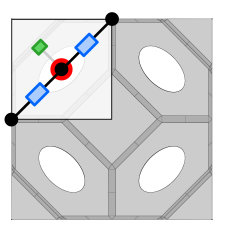

$4 \times \frac{1}{4} \times$

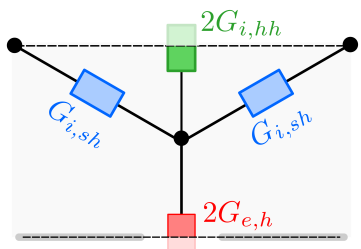

(d)
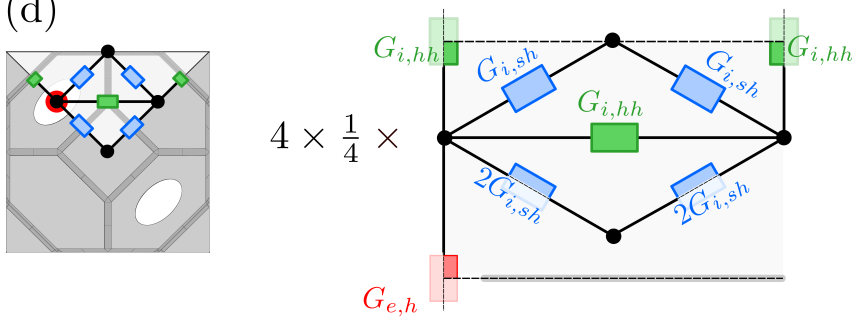

Figure 15. Conductance pore-networks associated to each PUC configuration.
Table I. Effective electrical conductivity $\sigma_{e}$ for each PUC configuration (Fig. 7).

\begin{tabular}{|c|c|}
\hline PUC & $\sigma_{e}$ \\
\hline \hline$K_{0}$ & $2 D_{b}^{-1}\left[2 G_{1} G_{2}+G_{e h}\left(G_{1}+G_{2}\right)\right]\left[G_{1}+G_{2}+2 G_{e h}\right]^{-1}$ \\
& $\begin{array}{c}\text { with } G_{1}=\left(G_{i, s h}^{-1}+2 G_{e s}^{-1}\right)^{-1} \\
\text { and } G_{2}=2\left(G_{i, s h}+G_{i, h h}\right)\end{array}$ \\
\hline$K_{1}$ & $4 D_{b}^{-1}\left\{2 G_{e s}^{-1}+G_{i, s h}^{-1}+\left[2 G_{i, h h}+2 G_{i, s h}\right]^{-1}\right\}^{-1}$ \\
\hline$K_{2}$ & $D_{b}^{-1}\left\{\left[2 G_{e h}\right]^{-1}+\left[2\left(G_{i, s h}+G_{i, h h}\right)\right]^{-1}\right\}^{-1}$ \\
\hline$K_{3}$ & $D_{b}^{-1}\left\{G_{e h}^{-1}+\left[\frac{3}{2}\left(G_{i, s h}+G_{i, h h}\right)\right]^{-1}-1\right\}^{-1}$ \\
\hline
\end{tabular}

Table II. Coefficients $n_{w}$ for various lattices and weakly disordered foam. Note that for SC, BCC or Kelvin lattices, $n_{w}$ is isotropic.

\begin{tabular}{|c||c|c|c|c|}
\hline Structure & SC & BCC $(\phi \lesssim 0.88)$ & BCC (Kelvin) & disordered \\
\hline$N_{v}$ & 6 & 8 & 14 & $2(n+1)$ \\
\hline$n_{w}$ & 1 & 2 & 4 & $\approx \frac{n}{2}$ \\
\hline
\end{tabular}

The mean local conductance $\bar{G}$ is iteratively calculated from [14, 16]:

$$
\frac{1}{\bar{G}+n \bar{G}}=\sum_{i} \frac{x_{i}}{G_{i}+n \bar{G}}
$$

with $x_{i}$ the fraction of local inter-pore electrical conductance $G_{i}$ and $n=\frac{N_{v}}{2}-1$.

The macroscopic effective conductivity $\sigma_{e}$ is then deduced from the mean local conductance $\bar{G}$,

$$
\sigma_{e}=n_{w} \bar{G} / D_{b}
$$

where the coefficient $n_{w}$ depends on the structure of the porous medium (Tab. II).

Note that when the fraction of open windows $x_{o w}$ is close to the percolation threshold, it is necessary to modify these equations to consider the structure of the openpore space as shown in [9].
[1] D. L. Johnson, J. Koplik, and R. Dashen, J. Fluid Mech. 176, 379 (1987).

[2] M. Biot, J. Acoust. Soc. Am. 28, 168 (1956).

[3] R. Venegas and C. Boutin, J. Fluid Mech. 828, 135 (2017).

[4] R. J. S. Brown, Geophysics 45, 1269 (1980).

[5] D. L. Johnson and P. N. Sen, Physical Review B 24, 2486 (1981).

[6] M.-Y. Zhou and P. Sheng, Phy Rev B 39, 12027 (1989).

[7] I. Cantat, S. Cohen-Addad, F. Elias, F. Graner, R. Höhler, O. Pitois, F. Rouyer, and A. Saint-Jalmes, Foams: structure and dynamics (Oxford University Press, 2013).

[8] C. Gaulon, J. Pierre, C. Derec, L. Jaouen, F.-X. Bécot, F. Chevillotte, F. Elias, W. Drenckhan, and V. Leroy,
Appl. Phys. Lett. 112, 261904 (2018).

[9] V. Langlois, V. H. Trinh, C. Lusso, C. Perrot, X. Chateau, Y. Khidas, and O. Pitois, Phys. Rev. E 97, 053111 (2018).

[10] V. Trinh, V. Langlois, J. Guilleminot, C. Perrot, O. Pitois, and Y. Khidas, Materials and Design 162, 345 (2019).

[11] J. Lee and I. Jung, Applied Acoustics 151, 10 (2018).

[12] H. L. Frisch, J. M. Hammersley, and D. J. A. Welsh, Phys. Rev. 126, 949 (1962).

[13] I. Fatt, Petroleum Transactions, AIME 207, 141 (1956).

[14] S. Kirkpatrick, Rev. Mod. Phys. 45, 574 (1973).

[15] L. M. Schwartz, N. Martys, D. P. Bentz, E. J. Garboczi, and S. Torquato, Phys. Rev. E 48, 4584 (1993). 
[16] P. Adler, Porous media : geometry and transports (Butterworth/Heinemann, 1992).

[17] R. A. Sampson, Philos. T. Roy. Soc. A. 182, 449 (1891).

[18] J. E. Hall, J. Gen. Physiol. 66, 531 (1975).

[19] A. M. Rosenfeld and R. S. Timsit, Q. Appl. Math. 39, 405 (1981).

[20] G. Windred, Journal of the Franklin Institute 231, 547 (1941).

[21] R. Holm, The contact resistance. General theory, edited by S.-V. B. Heidelberg (Springer, 1958).

[22] J. Newman, J. Electrochem. Soc. 113, 501 (1966).

[23] S. Sahu and M. Zwolak, Phys. Chem. Chem. Phys. 20, 4646 (2018).

[24] S. Sahu and M. Zwolak, Phys. Rev. E 98, 012404 (2018).

[25] C. Perrot, R. Panneton, and X. Olny, J. Appl. Phys. 101, 113538 (2007).

[26] C. Stubenrauch, A. Menner, A. Bismarck, and W. Drenckhan, Angewandte Chemie 57, 10024 (2018).

[27] G. Lutzweiler, J. Barthes, G. Koenig, H. Kerdjoudj,
J. Mayingi, F. Boulmedais, P. Schaaf, W. Drenckhan, and N. Vrana, ACS Appl. Mater. Interfaces 11, 19819 (2019).

[28] R. Panneton and X. Olny, J. Acoust. Soc. Am. 119, 2027 (2006).

[29] D. L. Johnson, T. J. Plona, C. Scala, F. Pasierb, and H. Kojima, Physical Review Letters 49, 1840 (1982).

[30] D. L. Johnson, D. L. Hemmick, and H. Kojima, Journal of Applied Physics 76, 104 (1994).

[31] P. Leclaire, L. Kelders, W. Lauriks, M. Melon, N. Brown, and B. Castagnède, J. Appl. Phys. 80, 2009 (1996).

[32] J. L. Auriault, C. Boutin, and C. Geindreau, Homogenization of coupled phenomena in heterogenous media, edited by U. John Wiley \& Sons, Hoboken, Vol. 1 (Wiley Online Library, 2009).

[33] M. Avellaneda and S. Torquato, Phys. Fluids A-Fluid 3, 2529 (1991). 